\title{
Low-Overhead Dominating Set based Algorithms for Maximizing Lifetime in Wireless Sensor Networks
}

\author{
Jian Ma and Lionel M. Ni \\ Department of Computer Science and Engineering \\ Hong Kong University of Science and Technology \\ Clear Water Bay, Kowloon, Hong Kong \\ \{majian,ni\}@cse.ust.hk
}

\begin{abstract}
In wireless sensor networks, connected dominating set (CDS) has become a key technique to identify redundant nodes and extend network lifetime. Most CDS-based algorithms focus on building the minimal CDS to find as many redundant nodes as possible. However, role rotation is not considered so that the dominating nodes will run out of energy much faster than the non-dominating nodes. Existing CDS-based algorithms for maximizing network lifetime rely on the up-to-date remaining energy level (REL) information within h-hop neighborhood to rotate node roles iteratively. Furthermore, global time synchronization is required to synchronize every round. The overhead on REL updating and time synchronization can lead to energy waste and packet collision. In this paper, we first propose a randomized rotation algorithm, which can totally avoid REL updating. Then, dominating node history is added as an enhancement to further extend network lifetime. Finally, we propose a broadcast-based synchronization mechanism to reduce the synchronization overhead and assist dominating node selection. Extensive simulations show that our proposed algorithm can significantly reduce overhead without sacrificing network lifetime.
\end{abstract}

Index Terms - Connected Dominating Set, Network Lifetime, Topology Control, Wireless Sensor Networks

\section{INTRODUCTION}

Recent development of wireless sensor networks (WSNs) bridges the gap between the physical and digital world [1]. Self-organizing a large number of tiny sensor nodes and aggregating a large amount of sensory data, WSNs greatly extend our ability to instrument with the physical world. WSNs have been applied in a wide range of applications such as environmental monitoring and battlefield surveillance.

Sensor nodes are usually battery-powered with limited computation capacity and communication bandwidth. Since typical WSN applications only require lightweight computation and low-rate communication, energy becomes the scarcest resource that determines the network lifetime of deployed WSNs. Thus, energyefficiency is one of the key problems for WSNs.

$1-4244-1455-5 / 07 / \$ 25.00$ C2007 IEEE
As WSNs are always densely deployed, an important approach to save energy is to turn off redundant nodes without affecting the running WSN application. Borrowed from graph theory, dominating set (DS) is a useful technique to figure out redundant nodes. In a graph, a set of vertices is dominating if every vertex in a graph is either in the set or adjacent to a vertex in the set. If the subgraph formed by a DS is connected, the set is called connected dominating set (CDS). CDS technique has been applied in WSNs in following ways. First, CDS-based topology control algorithms [2] use dominating nodes to construct the communication infrastructure in a WSN. Only dominating nodes need to maintain routing tables and forward packet. When a non-dominating node wants to send its packets, it just needs to send the packets to its neighboring dominating nodes. Furthermore, since dominating nodes can buffer the incoming packets for sleeping non-dominating nodes, non-dominating nodes can spend most of their time in sleeping. By differentiating non-dominating nodes with dominating nodes, we can save a lot of energy on non-dominating nodes. Second, to fight against the broadcast storm problem [3], CDS-based broadcast algorithms [4] select the dominating nodes as forwarding nodes so that non-dominating nodes are free from forwarding broadcast packets. Compared with CDSbased topology control algorithms, CDS-based broadcast algorithms can exploit the broadcast history to further reduce the size of CDS. Third, CDS-based scheduling algorithms [5] turn non-dominating nodes into sleeping while maintaining the full coverage of sensing fields. However, connectivity in DS is not always required in coverage problem. We will focus on CDS in this paper.

As CDS-based approaches [6-9] figure out nondominating nodes as redundant nodes to save energy, it is desired to find as many non-dominating nodes as possible. This leads to the well-known minimal $C D S$ (MCSD) problem, which has been proved to be NPhard [10]. Many heuristic-based algorithms have been proposed to construct MCDS in mobile ad hoc networks or WSNs. However, network lifetime is not con- 


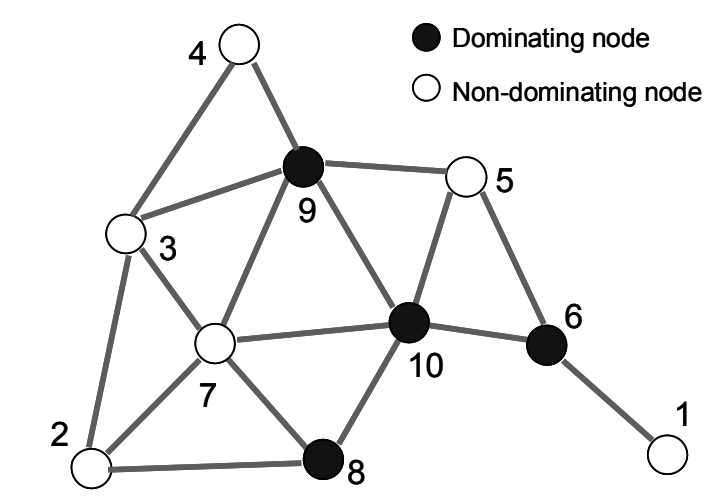

Figure 1. Rule $k$ algorithm.

sidered in those algorithms as the dominating nodes will not change their roles and run out of energy much faster than the non-dominating nodes. Several CDSbased algorithms [11-14] have been proposed to extend network lifetime by using some role rotation mechanisms, in which a node will iteratively adjust its role based on up-to-date residual energy level (REL) information within $h$-hop neighborhood. Furthermore, global time synchronization [15] is required to synchronize every round. The overhead on updating REL and time synchronization can consume a lot of energy, especially for large $h$ value and short update period. High overhead can also lead to difficulties in schedule update period and severe packet collisions. In addition, a large $h$ value can make the corresponding algorithms less responsive to changes as a node can be affected by any of its $h$-hop neighbors.

In this paper, we propose a series of low-overhead CDS-based algorithms, which can significantly reduce the overhead in existing algorithms while still achieving the same order of network lifetime. First, exploiting randomness to balance the duty of being dominating nodes among all the nodes, we propose a randomized role rotation algorithm to avoid REL updating. Then, to further extend the network lifetime, the randomized algorithm is enhanced by adding dominating node history into consideration. Finally, we propose a broadcast-based synchronization mechanism to reduce the overhead of synchronization and assist dominating node selection.

The rest of the paper is organized as follows. We discuss related topology control algorithms in Section II. Section III defines the maximum-lifetime CDS scheduling problem. Our low-overhead role rotation algorithms are detailed in Section IV. The broadcastbased synchronization mechanism is proposed in Section 0 . Section VI evaluates our proposed algorithm by extensive simulations. Finally, we give our conclusion and list future work in Section VII.

\section{RELATED WORK}

Guha and Khuller [10] proved that the MCDS problem is an NP-hard problem and proposed a centralized approximation algorithm. In the approximation algorithm, a non-dominating node can have two states COVERED and UNCOVERD. The non-dominating nodes that have a neighboring dominating node are in COVERED state. All the other non-dominating nodes are in UNCOVERED state. The algorithm greedily reduces the number of UNCOVERED nodes until every UNCOVERD node becomes either a COVERED node or a dominating node. However, centralized algorithms are not suitable for WSNs due to the extremely high cost of collecting global network topology.

Many distributed heuristic-based algorithms have been proposed for the MCDS problem. Wu and Li [2] have proposed a marking scheme to construct CDS in ad hoc networks. Each node with two directly unconnected neighbors is marked as a dominating node. Two pruning rules are used to reduce the size of constructed CDS. According to pruning rule 1, a dominating node can be unmarked if all of its neighbors are also neighbors of another neighbor that has a higher priority. According to pruning rule 2, a dominating node can become unmarked if all of its neighbors are also neighbors of either of two other neighbors that are connected and have higher priorities. Dai and $\mathrm{Wu}$ [6] extended the rule 1 and 2 to a more general pruning rule $k$ : a dominating node becomes a non-dominating node if all of its neighbors are also neighbors of any one of $k$ other nodes that are connected and have higher priorities. In other words, those $k$ nodes form a local CDS that dominates the neighbor set. The priority of a node is defined as its unique ID so that a node with larger ID will have higher priority to become a dominating node. To make the algorithm localized, only higher-priority nodes with $h$-hop neighborhood are considered to form a local CDS.

An example of Rule $k$ is illustrated in Figure 1. We take node 7 as an example. The set of higher-priority nodes includes node 8,9 , and 10 . This set is connected, and it covers the remaining three neighbors. According to Rule $k$ algorithm, node 7 will be a non-dominating node. In contrast, when node 6 is evaluated, its set of higher-priority neighbors just includes node 10 . Since one of its neighbors, node 1 , is not connected to node 10 , node 6 has to be a dominating node.

Rule $k$ algorithm becomes the foundation of many CDS-based algorithms due to its flexibility in terms of the size of neighborhood (the $h$ value) and the priority definition. Stojmenoic et al. [4] extended Rule $k$ algorithm by assuming that every node knows its accurate location through localization algorithms [16]. Each 


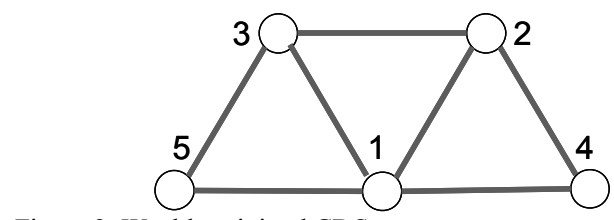

Figure 2. Weakly minimal CDS

node just needs to maintain a list of its neighbors and their geographic positions so that the overhead of collecting neighborhood information is reduced. Besides reducing overhead, several algorithms are proposed to further optimize the size of constructed CDS. Wu et al. [11] introduced node degree (ND) into the priority definition, in which a node with higher ND will have higher priority. $\mathrm{Wu}$ et al. [7] introduced an iterative solution, which iteratively runs Rule $k$ to reduce the size of constructed CDS round by round. Ma et al. [8] allowed a node to wait for its lower-priority neighbors before making its own decision. Wu and Dai [9] have proposed a general coverage condition, which covers all the above algorithms as its special cases. However, network lifetime is not considered in the above algorithms as the selected dominating nodes will not change their roles and run out of energy much faster than the non-dominating nodes.

Role rotation is essential for prolonging network lifetime in WSNs. Wu et al. [11] extended rule 1 and rule 2 with a role rotation mechanism, in which a node's priority depends on the RELs of itself and its neighbors. This REL-based mechanism can also be extended to Rule $k$ so that every node updates its REL information within $h$-hop neighborhood and computes its new role round by round. Chen et al. [12] proposed the Span protocol, in which a node becomes a dominating node if it has two neighbors that are not directly connected, indirectly connected via one intermediate dominating node, or indirectly connected via two dominating nodes. The priority of a node is computed from its REL and the number of its neighbor pairs that are not directly connected. Thus, Span needs topology and REL information within 3-hop neighborhood. Shaikh et al. [13] proposed the similar idea as Span and tested different combination of node degree and REL as new priority definitions. Bao and Garcia-LunaAceves [14] further added mobility into consideration. However, those REL-based algorithms require periodical update of REL information and global time synchronization in each round, which can introduce large overhead on energy consumption and increase packet collisions. Our proposed algorithms significantly reduce the two kinds of overhead without sacrificing network lifetime.

Moscibroda and Wattenhofer [17] proposed a DSbased maximum-lifetime algorithm, which constructs maximum number of disjoint dominating sets so that every DS candidate can become the active DS in turn to balance the total energy consumption. However, they only consider DS without the requirement of connectivity. Furthermore, the maximum-lifetime CDS schedule problem is not equivalent to the maximum disjoint CDS problem. We will discuss the difference between the two problems later.

\section{MAXIMUM-LIFETIME CDS Schedule Problem}

In this section, we will formally define the maximum-lifetime CDS schedule problem. The network is modeled as a general undirected graph $G=(V, E)$. Thus, we do not rely on the unrealistic disk radio model [18]. The bidirectional communication links can be maintained by acknowledgement in MAC protocols [19] or link filtering in routing protocols [20]. Every node $v$ has a unique node ID as $\operatorname{ID}(v)$. We want to balance the responsibility of becoming dominating nodes to all the nodes.

To simplify the problem, we assume the period of every round in role rotation is fixed. The initial REL of every node is $E$ joule. The workload on dominating nodes is homogeneously distributed to every dominating node for every round so that the REL of a dominating node will be reduced $d$ joule per round. Similarly, the REL of a non-dominating node will be reduced $d$, joule per round. Obviously, we have $d>d^{\prime}$. For a CDS $D$ and any node $v$, we can define the energy consumption function $e$ as

$$
e(D, v)=\left\{\begin{array}{c}
d, \text { if } v \in D \\
d^{\prime}, \text { otherwise }
\end{array}\right.
$$

A schedule $S$ is a sequence of CDS as $\left\{D_{1}, D_{2}, \ldots\right.$, $\left.D_{m}\right\}$, which satisfies the energy constraints on every node $v$ as

$$
\sum_{i=1}^{m} e\left(D_{i}, v\right) \leq E
$$

Since the lifetime of a WSN is defined as the length of period until the first node runs out of energy, the maximum-lifetime CDS schedule problem asks for the schedule $S$ with maximum length $m$ so that the maximum lifetime will be $m$ round. Intuitively, if $E=2$ and $d=1$, then every node can become a dominating node in at most two round.

In maximum-lifetime CDS schedule problem, we need to consider combinations of difference CDS rather than just minimizing single CDS. However, we still want to compute small-size CDS in each round. For any CDS $D$ is a schedule $S$, if there is another CDS $D^{\prime}$ satisfying $D^{\prime} \subset D$, we can generate a new schedule 


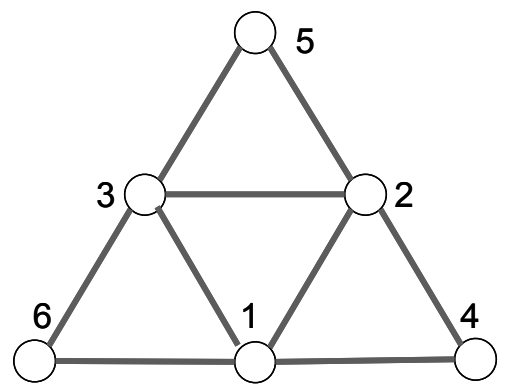

Figure 3. The difference between maximum-lifetime and maximum disjoint CDS problems.

$S^{\prime}$ by replacing $D$ with $D^{\prime}$, which still satisfies the energy constraints. Obviously, since $S^{\prime}$ consumes less energy than $S$, we may add more CDS into $S^{\prime}$ to get a better schedule. Thus, we define a weakly minimum $C D S$ (WMCDS) as a CDS with the property that if any one of its nodes is removed, the new set is not a CDS anymore. Obviously, a MCDS is a WMCDS, but a WMCDS may not be a MCDS. Take the graph in Figure 2 as an example, $\{1\}$ is a MCDS, $\{2,3\}$ is a WMCDS but not a MCDS. To get an optimal schedule, we want every CDS in the schedule to be a WMCDS.

If we list all the WMCDS in the graph $G$ as $W_{1}, W_{2}$, $\ldots, W_{k}$, we can transfer the maximum-lifetime CDS sequence problem into a linear programming optimization problem.

$$
\operatorname{maximize} \sum_{i=1}^{k} n_{i} \text { where } n_{i} \in 0 \bigcup Z^{+}
$$

$$
\text { subject to for } \forall v \in G, \sum_{i=1}^{m} n_{i} e\left(W_{i}, v\right) \leq E
$$

However, if we can list all the WMCDS, we can get the MCDS from them. Since MCDS is a NP-hard problem, listing all the WMCDS is also a NP-hard problem. The requirement of WMCDS also implies that the MCDS algorithms that apply pruning rules to reduce the size of CDS are still useful to generate WMCDS in each round.

As we mentioned before, a distributed approximation algorithm for maximum disjoint DS problem has been proposed in [17] to maximize lifetime. However, maximum disjoint CDS problem is not considered. Furthermore, the optimal partition for maximum disjoint CDS may not lead to the optimal schedule for maximum-lifetime CDS problem. An example is illustrated in Figure 3. Obviously, set $\{1,2\},\{2,3\}$, and $\{3$, $1\}$ are all the MCDS in this graph. We can easily prove that for any CDS $D$ in this graph, $|\mathrm{D} \cap\{1,2,3\}|>1$. In other words, the maximum number of disjoint CDS is just one. However, we can balance energy consumption among the $\operatorname{CDS}\{1,2\},\{2,3\}$, and $\{3,1\}$. If $d=1$, $d^{\prime}=0$, and $E=3$, the optimal partition of maximum disjoint CDS problem only has one CDS, which leads to a schedule with the lifetime of 3 rounds. In contrast, one of the optimal schedules for maximum-lifetime CDS problem is $\{\{1,2\},\{2,3\},\{3,1\},\{1,2\}\}$, which has longer lifetime.

For a WSN, each node will compute its new role in every round. The dominating nodes will broadcast its role announcement to its neighbors and form a communication infrastructure. After receiving one or several role announcements, a non-dominating node will choose one of its neighboring dominating nodes as its gateway node. The round period is predefined in deployments, which may highly depend on the surrounding environments. Generally, the more dynamic the environment is, the shorter the round period is.

Existing REL-based role rotation algorithms provide distributed solutions for the maximum-lifetime CDS scheduling problem. However, those algorithms require up-to-date REL information within $h$-hop neighborhood as well as global time synchronization, which can lead to energy waste and packet collision. We will propose algorithms to reduce the two types of overhead.

Besides topology control, aggregation [21] is another important approach to achieve energy-efficiency in WSNs by reducing the redundant messages in network communication.

\section{REL-INDEPENDENT Role Rotation ALgORIthmS}

After identifying the two types of overhead in role rotation algorithms, we aim at reducing the overhead of REL updating in this section. Exploiting randomness to balance the duty of being dominating nodes to all the nodes, we propose a randomized role rotation algorithm to avoid REL updating. To further extend the network lifetime, dominating node history is added as an enhancement for the randomized algorithm.

\section{A. Randomized Role Rotation}

The basic idea of REL-based rotation mechanism is to choose the CDS with high REL nodes as the next active CDS. However, according to the problem definition, the active order among CDS does not affect the schedule. Thus, it is not necessary to immediately select the CDS with high REL nodes in next round if we can select that CDS in a later round. If every WMCDS has the same chance to become an active CDS, We can distribute the responsibility of being dominating nodes to all the nodes by using random selection. In other words, REL-based approaches want to balance the energy consumption on all the nodes in each round, while our randomized mechanism wants to balance the energy consumption on all the nodes in long term. 


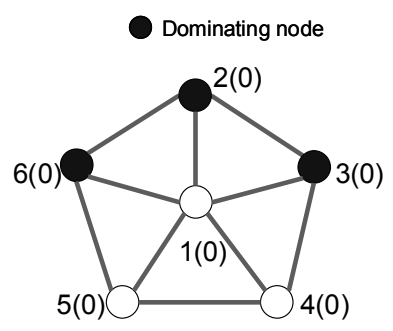

(a) Round 0

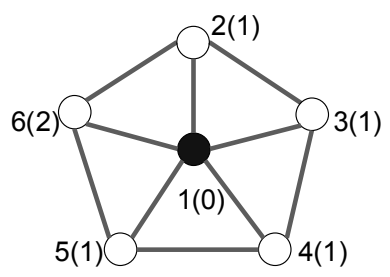

(c) Round 2 (b) Round 1

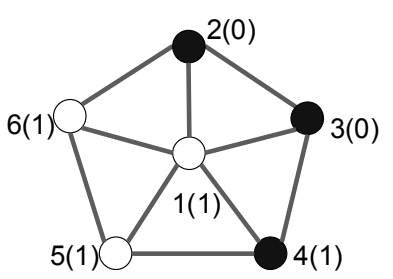

(d) Round 3

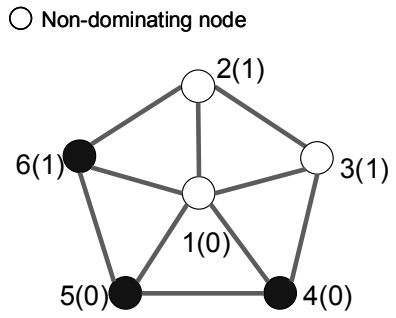

Figure 4. Randomized role rotation with history enhancement.

A straightforward idea is to generate random priority on every node. However, if a node randomly determines its priority on each round, it has to synchronize its newly generated priority to its neighbors. This can introduce the similar overhead caused by the periodical REL update. In order to eliminate the updating overhead, a node's priority must be known by its neighbors in advance. At the same time, we want to introduce dynamics into node priorities rather than just using the static node IDs. Thus, we want to define a well-defined priority sequence on each node, which can be computed by its neighbors without exchanging information on every round.

We denote the priority of node $v$ at round $t$ as $p_{t}(v)$. Every node takes its unique node ID as its initial priority such that $p_{0}(v)=\mathrm{ID}(v)$. We define a priority function $f_{p}$ such that $p_{t}(\mathrm{v})=f_{p}(\operatorname{ID}(v), t)$. If $f_{p}$ is predefined on all the nodes, node $v_{l}$ can compute the priority of node $v_{2}$ at any round $t$ as $f_{p}\left(\operatorname{ID}\left(v_{2}\right), t\right)$, if node $v_{l}$ already knows ID $\left(v_{2}\right)$.

As we want the priority function to generate randomness in different rounds, a good candidate is hash functions such as MD5. Currently the node ID defined in TinyOS [22] is 16-bit. We can express the round number as a 32-bit string and concatenate node ID with the round number to form a 48-bit input string. Thus, the priority function can be defined as

$$
f_{p}(\operatorname{ID}(v), t)=h\left(\operatorname{ID}(v)+{ }_{\mathrm{c}} t\right)
$$

where $h$ is the hash function we choose and $+_{\mathrm{c}}$ is the concatenation operation.

We can still use node ID to break the tie when $f_{p}$ generates two identical values, thus the priority of a node can be extended as a key where the first item is compared at first and then the second item is compared if needed.

$$
p_{t}(\mathrm{v})=\left(f_{p}(\mathrm{ID}(v), t), \operatorname{ID}(v)\right)
$$

After initially collecting $h$-hop topology information, a node will compute the keys for all its $h$-hop neighbors according to Equation (5) and follow Rule $k$ algorithm to determine its own role in each round.

\section{B. Dominating Node History Enhancement}

Even the randomized role rotation mechanism can well distribute the duty of becoming dominating nodes to all the nodes, it can shorten the network lifetime by randomly selecting a node with very little REL to become a dominating node. To take advantage of the benefit of REL-based approaches, we can add some REL-related information into consideration.

Since we do not want to introduce any REL overhead, we just consider the freely available REL-related information. As each dominating node will broadcast its role so that it can be selected by its neighbors as their gateway nodes, this kind of role announcement can give us some hints on RELs. Each node can record history of neighboring dominating nodes in previous $k$ rounds and adjust the priorities of these nodes according to the occurrence time in the history. A node with higher occurrence time will have lower priority. We denote function $o(v)$ as the occurrence time of node $v$ in the last $k$ rounds of dominating node history. The priority of node $v$ can be extended from Equation (5) as

$$
p_{t}(\mathrm{v})=\left(k-o(v), f_{p}(\mathrm{ID}(v), t), \operatorname{ID}(v)\right)
$$

An example of randomized role rotation algorithm with history enhancement is illustrated in Figure 4, which demonstrates the priority dynamics of node 1 and all its neighbors. Here we select $k=2$. The ID of any node $v$ is followed by its corresponding $o(v)$ value in the parentheses. At round 0 shown in Figure 4(a), the priorities of all the nodes are determined by the randomized priority function, and $\operatorname{WMCDS}\{2,3,6\}$ is selected. In round 1 shown in Figure 4(b), as the occurrence values of node 2,3 , and 6 become 1, WMCDS $\{4,5,6\}$ is selected. In round 2 shown in Figure 4(c), as node 1 has the smallest occurrence value, WMCDS $\{1\}$ is selected. Finally, in round 3 shown in Figure $4(d)$, since the occurrence values of node 2 and 3 are reset to $0, \operatorname{WMCDS}\{2,3,4\}$ is selected. From this example, we showed that the dominating node history really enhances the ability of the original randomized role rotation algorithm to balance the duty of becoming dominating nodes to different WMCDS.

\section{BROADCAST-BASED ROUND SYNCHRONIZATION}

After eliminating the requirements on REL updating, we focus on the round synchronization. Existing algorithms always assume that global time synchronization 


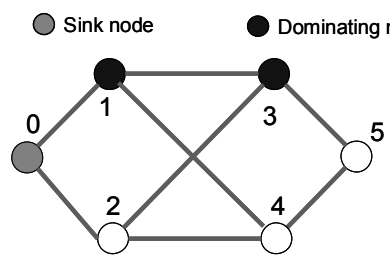

(a) Round $t$

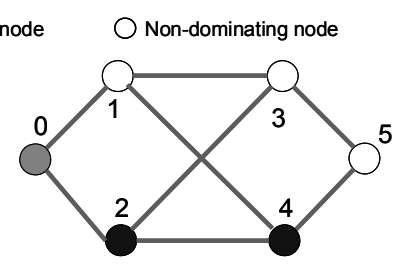

(b) Round $t+1$
Figure 5. Round synchronization.

is available so that every node will start a new round of role rotation in the same time. However, this kind of round synchronization introduces large overhead on energy consumption for large-scale sensor networks. Furthermore, synchronization inaccuracy can temporally break the communication backbone. An example is illustrated in Figure 5, where node 0 is the sink node. Since sink nodes usually have unlimited power supply, node 0 will always be a dominating node. As shown in Figure 5(a), node 1 and 3 are selected as dominating nodes at round $t$. Then, at round $t+1$, the responsibility will be rotated to node 2 and 4 . However, if there are some synchronization inaccuracies at the beginning of the round $t+1$, node 3 may become a non-dominating node before node 2 and 4 become dominating nodes. This causes two kinds of communication break. First, new non-dominating nodes such as node 3 cannot find their corresponding gateway nodes after switching from dominating roles. Second, old non-dominating nodes such as node 5 lose theirs existing gateway nodes without getting new gateway nodes. In other words, the switch between adjacent rounds is not smooth.

Rather than time synchronization, we will rely on broadcast from the sink node to synchronize rotation rounds. The sink node periodically broadcasts a round synchronization message, which contains the new round number, to all the nodes at the beginning of each round. A node starts the new round until it receives the corresponding round synchronization message. Since a dominating node continues to work until receiving the synchronization message, we can reduce the chance that a node loses its old gateway without getting new gateway node. Only the nodes that act as dominating nodes in the new round will forward the round synchronization message.

This synchronization mechanism can well eliminate the two kinds of communication break we mentioned before. First, for any new non-dominating node, due to our forwarding policy for round synchronization message, the source node of its received synchronization message must be a dominating node. Take the node 3 in Figure 5 as an example. Node 3 will only become a non-dominating node after the dominating node 2 rebroadcast the synchronization message. Second, for old non-dominating nodes, they can keep choosing their old gateway nodes to forward their packets because their old gateway nodes must be connected to the communication infrastructure in the new round. For example, in Figure 5(b), if node 3 becomes a nondominating node before node 4 switches to a dominating node, node 5 can still use node 3 to forward its packets since node 3 has already connected to the communication infrastructure in round $t+1$. Thus, our broadcast-based round synchronization mechanism can seamlessly switch between adjacent rounds.

TPSN synchronization protocol [15] creates a spanning tree of the network and then performs pairwise synchronization along the edges. Thus, every node sends one time synchronization packet. Exploiting the role announcements from dominating nodes, our broadcast-based round synchronization mechanism can significantly reduce the synchronization overhead.

\section{Evaluation}

In our simulations, sensor nodes are distributed in a square terrain with the side length of 100 meters. The communication range of every sensor node is $10 \mathrm{me}-$ ters. The sink node is placed in the center of the square. As random deployments can easily generate irregular topologies where some nodes have to constantly become dominating nodes no matter which rotation algorithm is applied, we choose uniform deployments in which the terrain is partitioned into grids with the side length of 10 meters and all the nodes are evenly divided amongst these grids. Sensor nodes are randomly deployed inside each grid. To consider different node densities, the number of deployed sensor nodes is changed from 300 to 2000 gradually. For each node density, the experiments are repeated 30 times and the average values are taken.

In our network topology, a node directly connects to all the nodes that reside in its communication range. If the generated placement cannot maintain network connectivity, this placement will be skipped.

We compare our randomized algorithm with two REL-based algorithms - Rule $k$ and Span. As we mentioned before, Rule $k$ and Span algorithm requires REL information within 2-hop and 3-hop neighborhood, respectively. As 3-hop neighborhood information can cause a lot of communication overhead and memory usage for resource-constrained sensor networks, we only consider a derivative of Span algorithm, which only relies on REL information within 2-hop neighborhood. We denote our purely randomized algorithm and randomized algorithm with history enhancement as Random and History, respectively.

In the default configuration for energy consumption, 


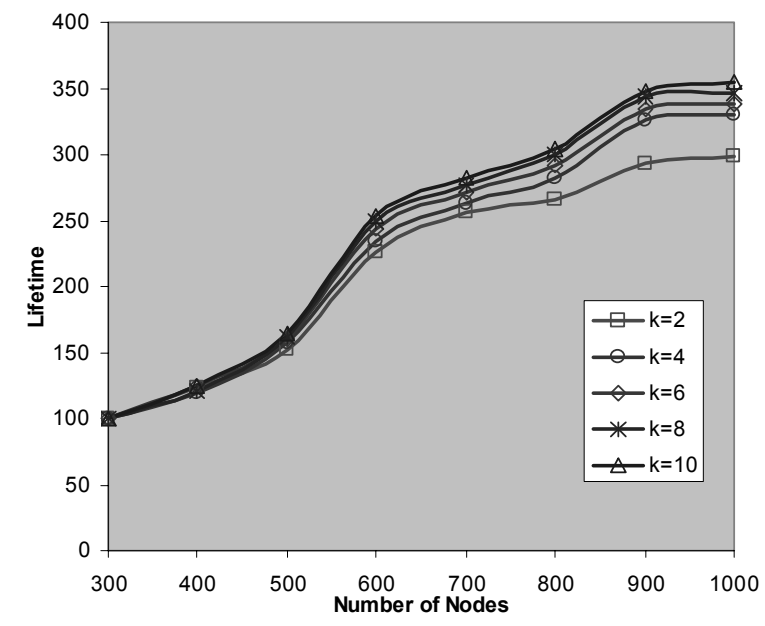

Figure 6. Network lifetime under different $k$.

we choose $E=1000, d=10$, and $d^{\prime}=1$. For simplicity, we assume each non-dominating node will consume 1 unit energy per time unit. The network lifetime is estimated in time units. This default configuration represents the scenario with short update period, where the minimum lifetime is 100 time units.

First we investigate the setting of history length $k$ for our History algorithm. As shown in Figure 6, we test $k$ from 2 to 10 with the step of 2 under different node densities. As the value of $k$ increases, the network lifetime also increases. However, the improvement of network lifetime is marginal after $k$ is larger than 4. A larger $k$ value also requires more memory usage for resource-constrained sensor nodes. Thus, we choose $k=10$ for our History algorithm in later simulations.

After finding the optimal setting of history length for our History algorithm, we compare all the selected algorithms in terms of network lifetime under the default configuration. As shown in Figure 7, when there are only 300 nodes, some nodes have to be dominating nodes all the time so that the lifetime reaches its minimum value as 100 time units. As node density increases, the chance of rotating nodes also increases. As there is a jump of lifetime improvement between 500 to 600 nodes for all the algorithms, we can regard 500 as a critical number to differentiate low density with high density. Among all the algorithms, Rule $k$ and History algorithms have the longest lifetime Span algorithm has much shorter lifetime because original Span algorithm relies on 3-hop neighborhood information. As the Random algorithm has the shortest lifetime, the enhancement of dominating node history for Random algorithm brings significant improvement over network lifetime.

We count three kinds of packets as overhead - REL updating, time synchronization, and dominating node

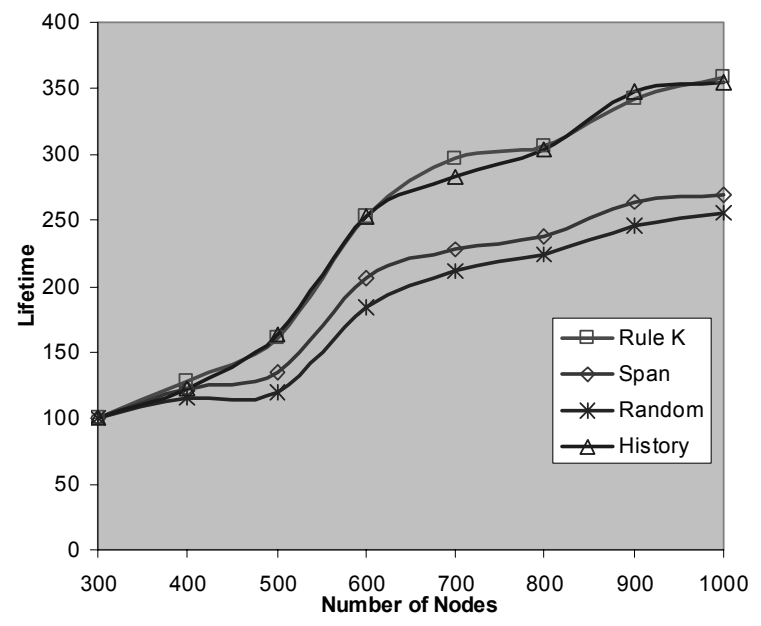

Figure 7. The comparison of network lifetime.

announcements. The number of packets for REL updating depends on the size of neighborhood where REL information is updating. As Random and History algorithms exploit dominating node announcements to synchronize different rounds, they only introduce overhead on dominating node announcements. The other algorithms need both time synchronization and dominating node announcements. The number of dominating node announcements is equivalent to the total number of dominating nodes in all the rounds. To normalize the overhead under different node density and network lifetime, we choose the number of overhead packets for each node per round as the metric. Thus, this metric is independent of the length of update period. The comparison of overhead for all the algorithms is shown in Figure 8. Our Random and History algorithms can significantly reduce the overhead in other algorithms.

As the default configuration represents the scenario with short update period, we consider the scenario with long update period now. We adjust the update period to be 5 time units such that $d=50$ and $d^{\prime}=5$ in the new configuration. The comparison of lifetime for all the algorithms is demonstrated in Figure 9. Compared with the result of default configuration shown Figure 7, the order of the algorithms keeps the same. Random algorithm suffers from longer update period because Random algorithm prefers a large number of update rounds to balance all the nodes through randomness. The lifetime of Rule $k$ and History algorithm is slightly shorten as longer update period makes the period scheduling more coarse-grained. For example, when $d^{\prime}=1$, a node with the REL of 2 energy units can be a nondominating node for 2 more rounds. However, when $d^{\prime}=5$, the node cannot even be a non-dominating node for the next rounds. Thus, the lifetime of this node is reduced by 2 time units in this example. 


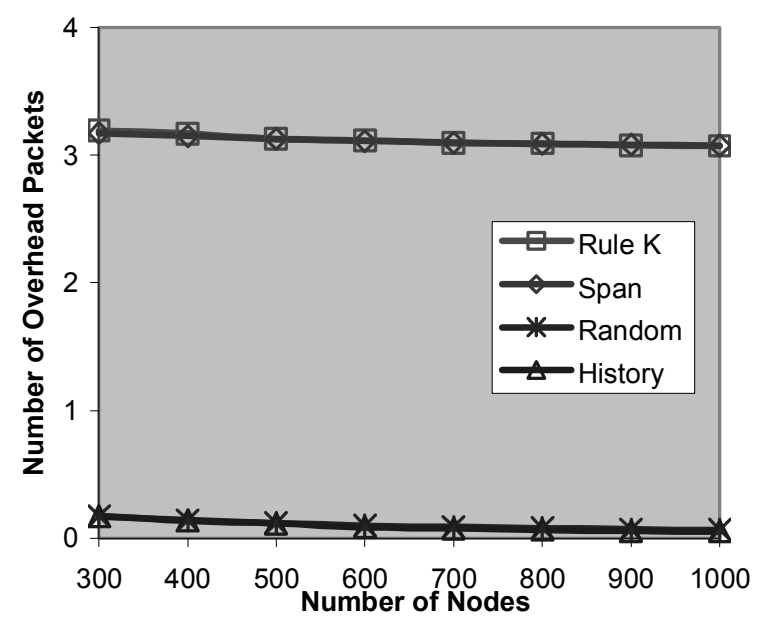

Figure 8 . The comparison of overhead.

Finally, we take insight in the impact of the ratio between energy consumption of dominating nodes and non-dominating nodes on network lifetime. In the default configuration, the ratio is equal to 10 . We further increase $d$ to 20 in new configuration and evaluate the network lifetime when the number of nodes is 500 and 600 , respectively. As we showed before, 500 is the critical number to differentiate low and high node density. The comparison between the two configurations in deployments with 500 and 600 nodes are shown in Figure 10(a) and Figure 10(b), respectively. As lifetime nearly halves for every algorithm, it shows that $d$ is the dominating factor for energy consumption when the ratio between $d$ and $d^{\prime}$ is high.

\section{Conclusions And Future Work}

In this paper, after defining the maximum-lifetime CDS scheduling problem, we have proposed a series of low-overhead role rotation algorithms. Compared with

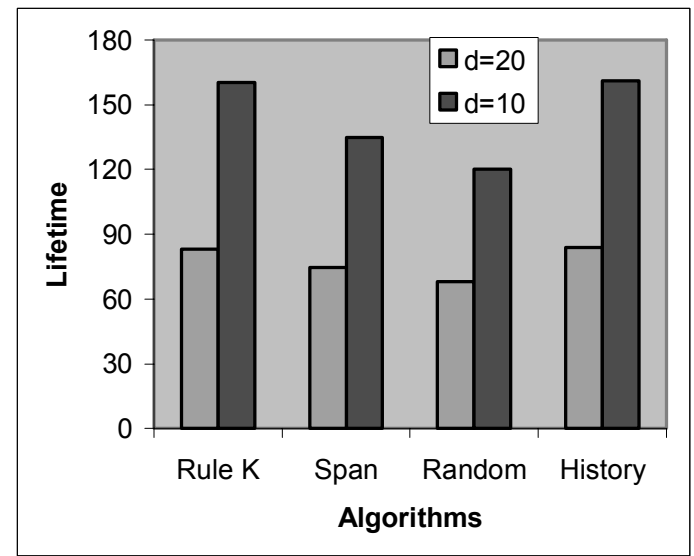

(a) 500 nodes

Figure 10. Different ratio between $d$ and $d^{\prime}$.

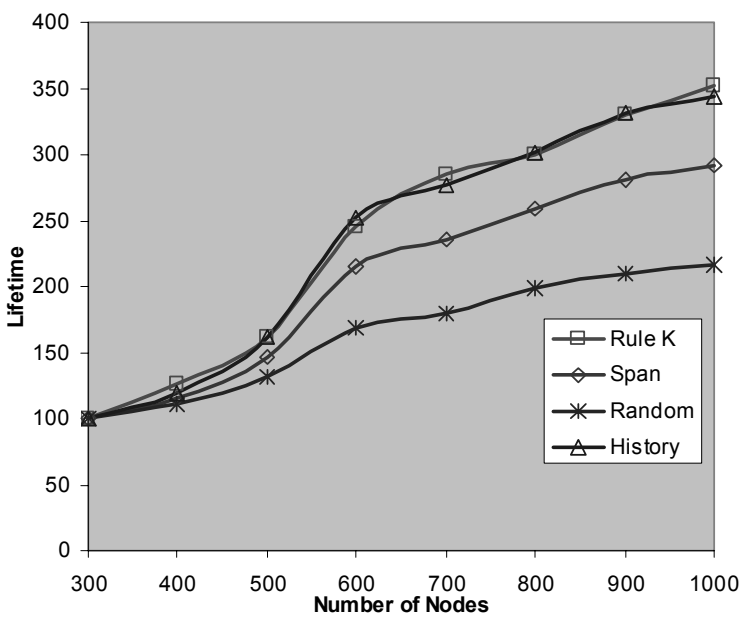

Figure 9. The comparison of network lifetime with longer update period.

existing algorithms, our algorithms can totally avoid the overhead of REL updating and significantly reduce the overhead of time synchronization, while still achieving the same order of network lifetime.

We are considering some possible extensions for the proposed algorithms. We can take randomized algorithms first and turn to REL-based algorithms when the RELs of some nodes are below certain threshold. We will also adopt some lightweight randomized priority functions, which needed much less computation than hash functions.

\section{ACKNOWLEDGMENT}

This research was supported in part by Hong Kong RGC Grant HKUST6183/05E, the Key Project of China NSFC Grant 60533110, the National Basic Research Program of China (973 Program) under Grant No. 2006CB303000, the Key Project of Guangzhou Municipal Government Guangdong/Hong Kong Criti-

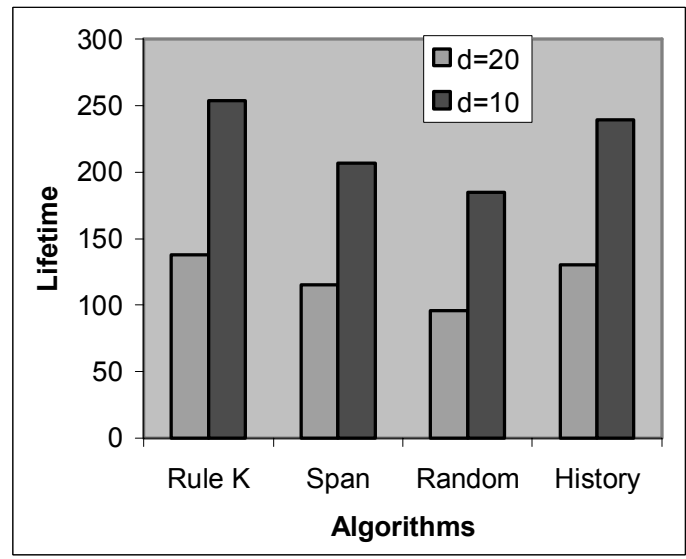

(b) 600 nodes 
cal Technology Grant 2006Z1-D6131 and the HKUST Nansha Research Fund NRC06/07.EG01.

\section{REFERENCE}

[1] I. F. Akyildiz, W. Su, Y. Sankarasubramaniam, and E. Cayirci, "A survey on sensor networks," IEEE Communications Magazine, vol. 40, no. 8, pp. 102-114, 2002.

[2] J. Wu and H. Li, "On calculating connected dominating set for efficient routing in ad hoc wireless networks," in Proceedings of the Third International Workshop on Discrete Algorithms and Methods for Mobile Computing and Communications, 1999.

[3] Y. C. Tseng, S. Y. Ni, Y. S. Chen, and J. P. Sheu, "The broadcast storm problem in a mobile ad hoc network," Wireless Networks, vol. 8, no. 2-3, pp. 153-167, 2002.

[4] I. Stojmenovic, M. Seddigh, and J. Zunic, "Dominating sets and neighbor elimination-based broadcasting algorithms in wireless networks," IEEE Transactions on Parallel and Distributed Systems, vol. 13, no. 1, pp. 14-25, 2002.

[5] J. Carle and D. Simplot-Ryl, "Energy-efficient area monitoring for sensor networks," Computer, vol. 37, no. 2, pp. 40-46, 2004.

[6] F. Dai and J. Wu, "Distributed dominant pruning in ad hoc networks," in Proceedings of the IEEE International Conference on Communications, 2003.

[7] J. Wu, F. Dai, and S. Yang, "Iterative local solutions for connected dominating set in ad hoc wireless networks," in Proceedings of the Second IEEE International Conference on Mobile Adhoc and Sensor Systems Conference, 2005.

[8] J. Ma, M. Gao, Q. Zhang, L. M. Ni, and W. Zhu, "Localized low-power topology control algorithms in IEEE 802.15.4-based sensor networks," in Proceedings of the Twenty-Fifth IEEE International Conference on Distributed Computing Systems, 2005.

[9] J. Wu and F. Dai, "A generic distributed broadcast scheme in ad hoc wireless networks," IEEE Transactions on Computers, vol. 53, no. 10, pp. 13431354, 2004.

[10] S. Guha and S. Khuller, "Approximation algorithms for connected dominating sets," Algorithmica, vol. 20, no. 4, pp. 374-387, 1998.

[11] J. Wu, F. Dai, M. Gao, and I. Stojmenovic, "On calculating power-aware connected dominating sets for efficient routing in ad hoc wireless networks," Journal of Communications and Networks, vol. 4, no. 1, pp. 59-70, 2002.

[12]B. Chen, K. Jamieson, H. Balakrishnan, and R. Morris, "Span: an energy-efficient coordination al- gorithm for topology maintenance in ad hoc wireless networks," in Proceedings of the Seventh Annual International Conference on Mobile Computing and Networking, 2001.

[13] J. A. Shaikh, J. Solano, and I. Stojmenovic, "New metrics for dominating set based energy efficient activity scheduling in ad hoc networks," in Proceedings of the Twenty-eighth Annual IEEE International Conference on Local Computer Networks, 2003.

[14]L. Bao and J. J. Garcia-Luna-Aceves, "Topology management in ad hoc networks," in Proceedings of the Fourth ACM International Symposium on Mobile Ad Hoc Networking and Computing, 2003.

[15] S. Ganeriwal, R. Kumar, and M. B. Srivastava, "Timing-sync protocol for sensor networks," in Proceedings of the First International Conference on Embedded Networked Sensor Systems, 2003.

[16] M. Li and Y. Liu, "Rendered path: range-free localization in anisotropic sensor networks with holes," in Proceedings of the Thirteenth Annual International Conference on Mobile Computing and Networking, 2007.

[17] T. Moscibroda, R. Wattenhofer, and A. Zollinger, "Topology control meets SINR: the scheduling complexity of arbitrary topologies," in Proceedings of the Seventh ACM international symposium on Mobile ad hoc networking and computing, 2006.

[18]D. Kotz, C. Newport, R. S. Gray, J. Liu, Y. Yuan, and C. Elliott, "Experimental evaluation of wireless simulation assumptions," in Proceedings of the Seventh International Symposium on Modeling, Analysis and Simulation of Wireless and Mobile Systems, 2004.

[19] W. Ye, J. Heidemann, and D. Estrin, "An energyefficient MAC protocol for wireless sensor networks," in Proceedings of the Twenty-First Annual Joint Conference of the IEEE Computer and Communications Societies, 2002.

[20] A. Woo, T. Tong, and D. Culler, "Taming the underlying challenges of reliable multihop routing in sensor networks," in Proceedings of the First International Conference on Embedded Networked Sensor Systems, 2003.

[21] Y. Liu and M. Li, "Iso-Map: energy-efficient contour mapping in wireless sensor networks," in Proceedings of the International Conference on Distributed Computing Systems, 2007.

[22] J. Hill, R. Szewczyk, A. Woo, S. Hollar, D. Culler, and K. Pister, "System architecture directions for networked sensors," in Proceedings of the Ninth International Conference on Architectural Support for Programming Languages and Operating Systems, 2000. 